\title{
Interface Generation and Compositional Verification in JavaPathfinder
}

\author{
Dimitra Giannakopoulou and Corina S. Păsăreanu \\ Carnegie Mellon University/NASA Ames Research Center, \\ Moffett Field, CA 94035, USA, \\ \{dimitra.giannakopoulou, corina.s.pasareanu\}@nasa.gov
}

\begin{abstract}
We present a novel algorithm for interface generation of software components. Given a component, our algorithm uses learning techniques to compute a permissive interface representing legal usage of the component. Unlike our previous work, this algorithm does not require knowledge about the component's environment. Furthermore, in contrast to other related approaches, our algorithm computes permissive interfaces even in the presence of non-determinism in the component. Our algorithm is implemented in the JavaPathfinder model checking framework for UML statechart components. We have also added support for automated assume-guarantee style compositional verification in JavaPathfinder, using component interfaces. We report on the application of the approach to interface generation for flight-software components.
\end{abstract}

\section{Introduction}

Component interfaces are a central concept in component-based software engineering. Although in current practice, interfaces typically describe the services that a component provides and requires at a purely syntactic level, the need has been identified for interfaces that document richer aspects of component behavior. Such extended interfaces are usually not provided, which makes their automatic generation an area of active research [10 10.

This paper addresses the automatic generation of interfaces that describe legal sequences of component calls. Such interfaces can serve as a documentation aid to application programmers, but can also be used by verification tools in checking that components are invoked correctly within a system. In fact, component interfaces are key for modular program analysis. They reduce the task of verifying a system consisting of a component and a client, to the more tractable task of verifying that the client satisfies the component's interface.

In previous work 6 6]6, we presented a framework based on learning, to perform automated assume-guarantee model checking of safety properties. To check that a system consisting of components $M_{1}$ and $M_{2}$ satisfies a safety property $P$, the framework automatically builds and refines assumptions $A$ for one of the components, for example $M_{1}$, to satisfy $P$, which it then tries to discharge on the other component, $M_{2}$. Although assumptions $A$ essentially constitute interfaces for component $M_{1}$, their generation relies on knowledge of component $M_{2}$.

M. Chechik and M. Wirsing (Eds.): FASE 2009, LNCS 5503, pp. 94-108, 2009.

(C) Springer-Verlag Berlin Heidelberg 2009 
Moreover, the focus of the framework was to compute assumptions that would allow to prove or disprove the property in the system, rather than assumptions that precisely document the behavior of a component.

The algorithm presented here for interface generation is also based on learning. However, in contrast to our work discussed above, it concentrates on the creation of precise component interfaces, irrespective of the component clients. By precise, we mean safe and permissive, as defined in [10]. An interface is safe if it accepts no illegal sequence of calls to the component. An interface is permissive if it includes all the legal sequences of calls to the component. Moreover, in [8], we presented an algorithm for generating what we call weakest assumptions in the context of Labeled Transition Systems. Weakest assumptions essentially constitute precise component interfaces. The difference of the current algorithm is that it is iterative, meaning that it can return partial results. Moreover, the approach in [8] required an expensive determinization step that we avoid here by dealing with the non-determinism in the component dynamically, during component analysis, as guided by counter-examples. Furthermore, our past experience, as well as other independent work [5], has indicated that the learning-based approach is more efficient for components that have relatively small interfaces.

Henzinger et al. also target the generation of safe and permissive interfaces in 10. Unlike our framework, their work based on abstraction techniques and it is only applicable to components that are visibly deterministic. The latter requires that the behavior of the component be deterministic with respect to the methods / actions in its communication interface (we will henceforth call the communication interface of a component its alphabet in order to avoid confusion with interface in this context). In the applications that we have been dealing with, this requirement proved too restrictive. For example, we often need to generate interfaces that focus on specific aspects of the component behavior, and that therefore include only a subset of the component's alphabet. Components that are visibly deterministic with respect to their full alphabet, typically lose this property when a subset of that alphabet is considered. Finally, Alur et al. 1] also use learning to synthesize interface specifications for abstracted Java components. However, their approach is heuristic-based, i.e., they do not always obtain precise interfaces.

We have implemented our algorithms in the JavaPathfinder (JPF) model checking framework for UML statechart components [1]. We have also added support for automated assume-guarantee style compositional verification in JPF, using component interfaces. JPF is an open source model checker for Java programs which, until now, provided no support for compositional verification.

The contributions of this work can be summarized as follows:

1. A novel algorithm for automated generation of precise component interfaces, also applicable to components that are not visibly deterministic

2. Implementation of our algorithm in the JPF open source model checker. In addition to interface generation, we have provided support for verification of safety properties expressed as finite state automata as well as assumeguarantee reasoning in JPF, where assumptions and guarantees are both 
expressed as finite-state automata. The implementation is freely available as JPF's compositional verification (cv) extension.

3. Case studies in the context of NASA applications that demonstrate the use of our algorithm in practice.

Related Work. The work closest to ours was discussed above. Several other approaches to automatic generation of component interfaces have been proposed in the literature. For example, Whaley et al. 19] use a combination of static and dynamic analyses to generate interfaces for Java components. Tkachuk et. al [18] use static analysis to obtain component abstractions, used as environments during modular analysis. Some approaches are based on extracting interfaces from sample execution traces [3. All these techniques generate approximate interfaces, as opposed to our work that aims at producing precise interfaces that provide correctness guarantees. Interface generation is related to compositional verification. In particular, assume-guarantee reasoning is a compositional approach that uses assumptions when reasoning about components in isolation [12 17/27] and component interfaces can be used as assumptions in this context. Finally, learning has also been used to generate models for formal verification [149]; those approaches do not address interface generation or compositional reasoning.

\section{Background}

We model software components using labeled finite state transition systems (LTSs), where transitions are labeled with component actions.

Let $\mathcal{A}$ ct be the universal set of observable actions and let $\tau$ denote a local action unobservable to a component's environment. Let $\pi$ denote a special error state, which models safety violations in the associated transition system.

LTSs. An LTS $M$ is a four-tuple $\left\langle Q, \alpha M, \delta, q_{0}\right\rangle$ where: $Q$ is a finite non-empty set of states; $\alpha M \subseteq \mathcal{A}$ ct is a set of observable actions called the alphabet of $M$; $\delta \subseteq Q \times(\alpha M \cup\{\tau\}) \times Q$ is a transition relation; and $q_{0} \in Q$ is the initial state.

Let $M=\left\langle Q, \alpha M, \delta, q_{0}\right\rangle$ and $M^{\prime}=\left\langle Q^{\prime}, \alpha M^{\prime}, \delta^{\prime}, q_{0}^{\prime}\right\rangle . M$ transits into $M^{\prime}$ with action $a$, denoted $M \stackrel{a}{\longrightarrow} M^{\prime}$, if $\left(q_{0}, a, q_{0}^{\prime}\right) \in \delta, Q=Q^{\prime}, \alpha M=\alpha M^{\prime}$, and $\delta=\delta^{\prime}$.

An LTS $M=\left\langle Q, \alpha M, \delta, q_{0}\right\rangle$ is non-deterministic if it contains $\tau$-transitions or if there exists $\left(q, a, q^{\prime}\right),\left(q, a, q^{\prime \prime}\right) \in \delta$ such that $q^{\prime} \neq q^{\prime \prime}$. Otherwise, $M$ is deterministic.

Traces. A trace $t$ of an LTS $M$ is a finite sequence of observable actions that label the transitions that $M$ can perform starting at its initial state, ignoring the $\tau$-transitions. For $\Sigma \subseteq \mathcal{A}$ ct, we use $t \uparrow \Sigma$ to denote the trace obtained by removing from $t$ all occurrences of actions $a \notin \Sigma$. For a set of traces $T$, $T \uparrow \Sigma=\left\{t \mid \exists t^{\prime} \in T . t^{\prime} \uparrow \Sigma=t\right\}$.

Parallel Composition. Parallel composition " $\|$ " is a commutative and associative operator: given LTSs $M_{1}=\left\langle Q^{1}, \alpha M_{1}, \delta^{1}, q_{0}^{1}\right\rangle$ and $M_{2}=\left\langle Q^{2}, \alpha M_{2}, \delta^{2}, q_{0}^{2}\right\rangle$, 
$M_{1} \| M_{2}$ is an LTS $M=\left\langle Q, \alpha M, \delta, q_{0}\right\rangle$, where $Q=Q^{1} \times Q^{2}, q_{0}=\left(q_{0}^{1}, q_{0}^{2}\right)$, $\alpha M=\alpha M_{1} \cup \alpha M_{2}$, and $\delta$ is defined as follows: (1) $M_{1}\left\|M_{2} \stackrel{a}{\longrightarrow} M_{1}^{\prime}\right\| M_{2}$ if $M_{1} \stackrel{a}{\longrightarrow} M_{1}^{\prime}$ and $a \notin \alpha M_{2}$, (2) $M_{1}\left\|M_{2} \stackrel{a}{\longrightarrow} M_{1}\right\| M_{2}^{\prime}$ if $M_{2} \stackrel{a}{\longrightarrow} M_{2}^{\prime}$ and $a \notin \alpha M_{1},(3) M_{1}\left\|M_{2} \stackrel{a}{\longrightarrow} M_{1}^{\prime}\right\| M_{2}^{\prime}$ if $M_{1} \stackrel{a}{\longrightarrow} M_{1}^{\prime}, M_{2} \stackrel{a}{\longrightarrow} M_{2}^{\prime}$, and $a \neq \tau$.

\section{Interface Generation}

In this section we define safe and permissive interfaces for software components and we describe our approach to synthesizing such interfaces automatically.

\subsection{Safe and Permissive Interfaces}

Let $M$ be a software component. For simplicity of presentation, we will first assume that $M$ includes an error state that expresses the undesired behavior of $M$ (for example, some assertion violations). Later in this section we will discuss the more general case where the component property is given as a separate (safety) automaton.

Let $\Sigma \subseteq \alpha M$ denote the communication alphabet of component $M$, i.e., the set of actions through which $M$ communicates with its environment. Our goal is to compute $M$ 's precise interface as a finite state automaton $A$ over $\Sigma$. As mentioned, we need to make sure that $A$ is both safe and permissive, as defined formally below.

Let us first define the legal and illegal languages of component $M$. A word $t \in \alpha M^{*}$ is illegal if it corresponds to some trace of $M$ that leads to error state $\pi$; otherwise, the word is legal. Then $\mathcal{L}_{\text {legal }}(M)$ denotes the set of legal words of $M$ and $\mathcal{L}_{\text {illegal }}(M)$ denotes the set of illegal words of $M$. Note that $\mathcal{L}_{\text {legal }}(M)$ and $\mathcal{L}_{\text {illegal }}(M)$ are complementary. Furthermore, note that, while illegal words correspond to actual traces in the component, legal words may also represent behavior that is never executed by the component (and hence could never lead to violations).

Definition 1. $A$ is a safe interface iff $\mathcal{L}_{\text {legal }}(A) \cap \mathcal{L}_{\text {illegal }}(M) \uparrow \Sigma=\emptyset$.

In other words, an interface is safe if it accepts no illegal words of $M$.

Definition 2. $A$ is a permissive interface iff $\mathcal{L}_{\text {legal }}(M) \uparrow \Sigma \subseteq \mathcal{L}_{\text {legal }}(A)$.

In other words, an interface is permissive if it accepts all legal words of $M$.

\subsection{Learning Interface Specifications with L*}

Our approach for learning interface specifications is illustrated in Figure 1, We use an off-the-shelf learning algorithm, $\mathrm{L}^{*}$ [4], to iteratively compute interface specification $A$ for $M$ that is both safe and permissive. $\mathrm{L}^{*}$ learns an unknown language (over a given alphabet) and produces a minimal deterministic finite state automaton that accepts it; the learning process is iterative and it uses a teacher that provides answers to queries and counterexamples to conjectures (for details on $\mathrm{L}^{*}$ see [4]). In our framework, the problem of answering queries and counterexamples is reduced to reachability problems, solved by a model checker. 


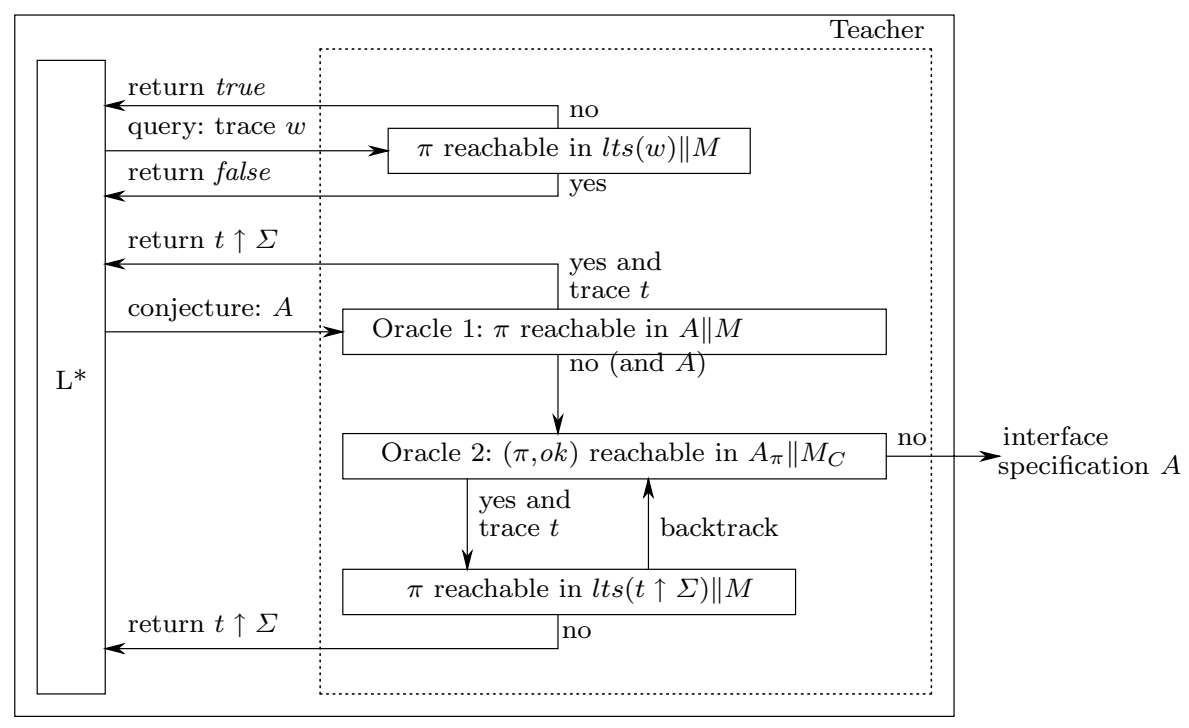

Fig. 1. Learning interface specifications with $\mathrm{L}^{*}$

Queries $\mathrm{L}^{*}$ is first used to repeatedly query $M$ to check whether or not, in the context of strings $w, M$ violates the property. This is equivalent with checking if an error state $\pi$ is reachable in $l t s(w) \| M$. Here $l t s(w)$ denotes an LTS over $\Sigma$ that accepts string $w$ (and its prefixes). The results of the queries are used by $\mathrm{L}^{*}$ to first make a "conjecture", i.e. it builds an automaton $A$ that accepts all the strings for the positive queries (the case error unreachable), and does not accept the strings for the negative queries (the case error reachable).

The conjectured automaton $A$ is then checked to make sure it is both safe and permissive. This is done with the help of a teacher that implements two oracles as described below.

Oracle 1 checks if $A$ is safe by checking whether $\pi$ is reachable in $A \| M$. If it is, then it means that $A$ is un-safe. The resulting counterexample $t$, projected on the interface alphabet $\Sigma$, is returned to $\mathrm{L}^{*}$ to refine its conjecture. If the error state is un-reachable, then it means $A$ is safe and we proceed to Oracle 2.

Oracle 2 checks if safe interface $A$ is also permissive, i.e. we want to check that $\mathcal{L}_{\text {legal }}(M) \uparrow \Sigma \subseteq \mathcal{L}_{\text {legal }}(A)$. This amounts to making sure that there are no words $w \in \Sigma^{*}$ such that $w \in \mathcal{L}_{\text {legal }}(M) \uparrow \Sigma \cap \mathcal{L}_{\text {illegal }}(A)$. This is equivalent to $w \in \mathcal{L}_{\text {illegal }}(A)$ and $\forall t \in \alpha M$ such that $w=t \uparrow \Sigma, t \in \mathcal{L}_{\text {legal }}(M)$.

We search for such words using a special reachability procedure performed on $A_{\pi} \| M_{C}$ (see pseudo-code in Figure 2). Here $A_{\pi}$ denotes the completion of $A$ with an error state, i.e. we complete each state with outgoing transitions to $\pi$, such that each state has outgoing transitions labeled with every action in $\Sigma$. Similarly, $M_{C}$ denotes the completion of $M$ with a special sink state. We 


\section{Oracle 2}

input: safe interface $A$;

begin

(1) Model-check $A_{\pi} \| M_{C}$ :

if $(\pi, o k)$ is reachable by trace $t$ then

(7) output: safe and permissive interface $A$;

end.

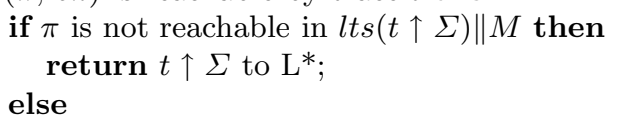

backtrack;

Fig. 2. Oracle 2

need these constructions to reason about traces in $\mathcal{L}_{\text {illegal }}(A)$ and $\mathcal{L}_{\text {legal }}(M)$, respectively. Note that $\mathcal{L}_{\text {illegal }}(A)=\mathcal{L}_{\text {illegal }}\left(A_{\pi}\right)$ and $\mathcal{L}_{\text {legal }}(M)=\mathcal{L}_{\text {legal }}\left(M_{C}\right)$. Note also that for Oracle 2 , since both $A_{\pi}$ and $M_{C}$ contain error states, we need to distinguish between the two in $A_{\pi} \| M_{C}$ (this was not necessary for queries and Oracle 1).

Given the above constructions, checking permissiveness reduces to checking reachability of states of the form: $(\pi, o k)$, were $\pi$ is an error state coming from $A_{\pi}$ and $o k$ denotes a non-error state in $M_{C}$. If such a combined state is found, then the trace $t$ leading to it may indicate that $A$ is not permissive, since $w=t \uparrow \Sigma$ leads to an error state in $A_{\pi}$ but it is legal in $M_{C}$ (and hence in $M$ ). However, due to non-determinism in $M$ (and hence in $M_{C}$ ), it may be the case that on another path, $t$ does lead to the error state. Even if this is not the case, there may exist other traces $t^{\prime}$ such that $w=t^{\prime} \uparrow \Sigma$ and $t^{\prime}$ leads to an error in $M_{C}$ on a different path (see Figure 3).

We check both these cases by performing a query on $t \uparrow \Sigma$. Note that we do not stop the state space exploration, but rather, we take trace $t$ that is returned, and we check if, in the context of $t \uparrow \Sigma, M$ violates its properties.

If the query returns true, then it means the interface is not permissive, and therefore $t \uparrow \Sigma$ is returned to $\mathrm{L}^{*}$ for refinement, and the learning process continues with more queries and eventually with a new conjecture.

If the query returns false, then $t$ does not correspond to a real counterexample. Model checking therefore ignores this state; it backtracks, and then continues its state space exploration. If no traces that satisfy the condition above exist, then indeed the conjectured automaton is also the most permissive interface, and therefore it is output to the user.

We note that every query is stored in the $\mathrm{L}^{*}$ memoized table, so the result of the query on the same trace $t \uparrow \Sigma$ later (when $A$ is the same) will be obtained directly (and faster) from the table.

Properties as safety automata. Assume now that $M$ does not have error states, and we want to generate an interface specification for ensuring a property $P$, given as a (deterministic) safety automaton, encoding all the desired behaviors of the component. Conversely, $P_{\pi}$ encodes all the un-desired behaviors of the 
State space of $A_{\pi} \| M_{C}$

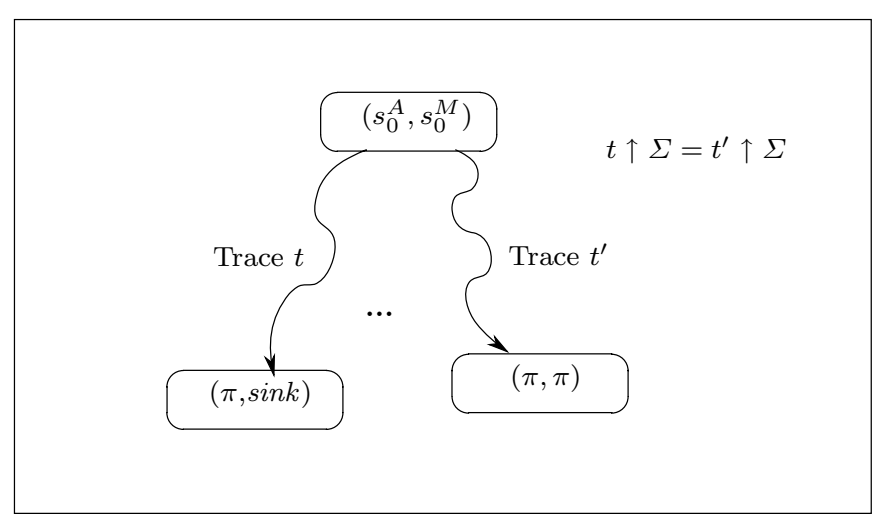

Fig. 3. Example for Oracle 2: dealing with non-determinism

component. The procedure described above will be exactly applicable to this case as well, if we treat $M \| P_{\pi}$ as $M$ above.

\subsection{Correctness and Termination}

We argue here the correctness and termination of our approach. To argue correctness, we first show that Oracle 1 (and similarly the queries) guarantee a safe interface while Oracle 2 guarantees a permissive interface; therefore, the teacher implemented by our approach is correct.

Proposition 1. Oracle 1 returns $A$ iff $\mathcal{L}_{\text {legal }}(A) \cap \mathcal{L}_{\text {illegal }}(M) \uparrow \Sigma=\emptyset$.

Proposition 2. Oracle 2 returns $A$ iff $\mathcal{L}_{\text {legal }}(M) \uparrow \Sigma \subseteq \mathcal{L}_{\text {legal }}(A)$.

Due to lack of space we omit the proofs here; they proceed by contradiction and follow the arguments given informally in the previous section.

Theorem 1. Given component finite state $M$ (that may include error states), the algorithm implemented by our approach terminates and it returns a safe and permissive interface $A$.

Proof. Correctness follows from the two propositions above. Termination follows from the correctness of $L^{*}$, which is guaranteed that, if it keeps receiving counterexamples, it will eventually terminate.

Discussion. As mentioned, in previous work we defined an algorithm for building safe and permissive interfaces for finite state components $[8$. That algorithm involves the determinization of $M$ (using the sub-set construction) that results in an exponential cost in computation time, regardless of the size of the interface specification. However, for components with small interfaces, the interface automaton is expected to be much smaller than the component itself. We address 
this problem by using $\mathrm{L}^{*}$, which builds incrementally automata with increasing size, finishing with the minimal deterministic automaton representing a safe and permissive interface.

We also note here that the approach of Henzinger et al. 10 can only handle components that are visibly deterministic, and therefore could not handle the case illustrated in Figure 3. On the other hand, the approach of Alur et al. 1] handles non-deterministic components, but it does not guarantee that the interface is permissive, since it only uses heuristics to implement what it amounts to Oracle 2 (called "superset query" in [1]). That work argues that the superset query can not be implemented efficiently, since it involves the determinization of component $M$. In our work we avoid an explicit determinization step of $M$. Instead, our approach deals with the non-determinism in the component dynamically (during model checking of the component) and only selectively (as guided by counterexamples).

\section{Compositional Verification in JPF}

\subsection{Java PathFinder}

Java PathFinder (JPF) [1] is an open-source verification framework developed by the RSE group at NASA Ames. It has been started as an explicit state model checker for Java byte-code. The focus of JPF is on finding bugs, such as concurrency related bugs (deadlocks, races, missed signals etc.), runtime related bugs (e.g. unhandled exceptions), etc. JPF can also check for violations of userspecified assertions that encode application specific requirements. JPF uses a variety of scalability enhancing mechanisms, such as user extensible state abstraction and matching, on-the-fly partial order reduction, configurable search strategies, and user definable heuristics (searches, choice generators).

\subsection{JPF's UML Statechart Extension}

JPF has recently been extended with a statechart modeling and analysis capability that allows Java modeling of UML state machines [15. Many UML development systems can produce code from diagrams, but this code is usually aimed at production systems, and is not suitable for software model checkers. The approach taken in JPF (Figure 4(left)) is based on a specific translation scheme from UML state charts into Java code that (a) is highly readable, (b) shows close correspondence between diagram and program, (c) provides a 1:1 mapping between model and program states, and (d) imposes few restrictions about aspects and actions that can be modeled.

The JPF Statechart extension is specialized to handle the obtained Java models more efficiently than random Java code. These Java models can be run in isolation, which corresponds to running them in the context of an external environment that may provide any input event at any stage (we will call this the universal environment). Alternatively, a guidance script may be provided by the 

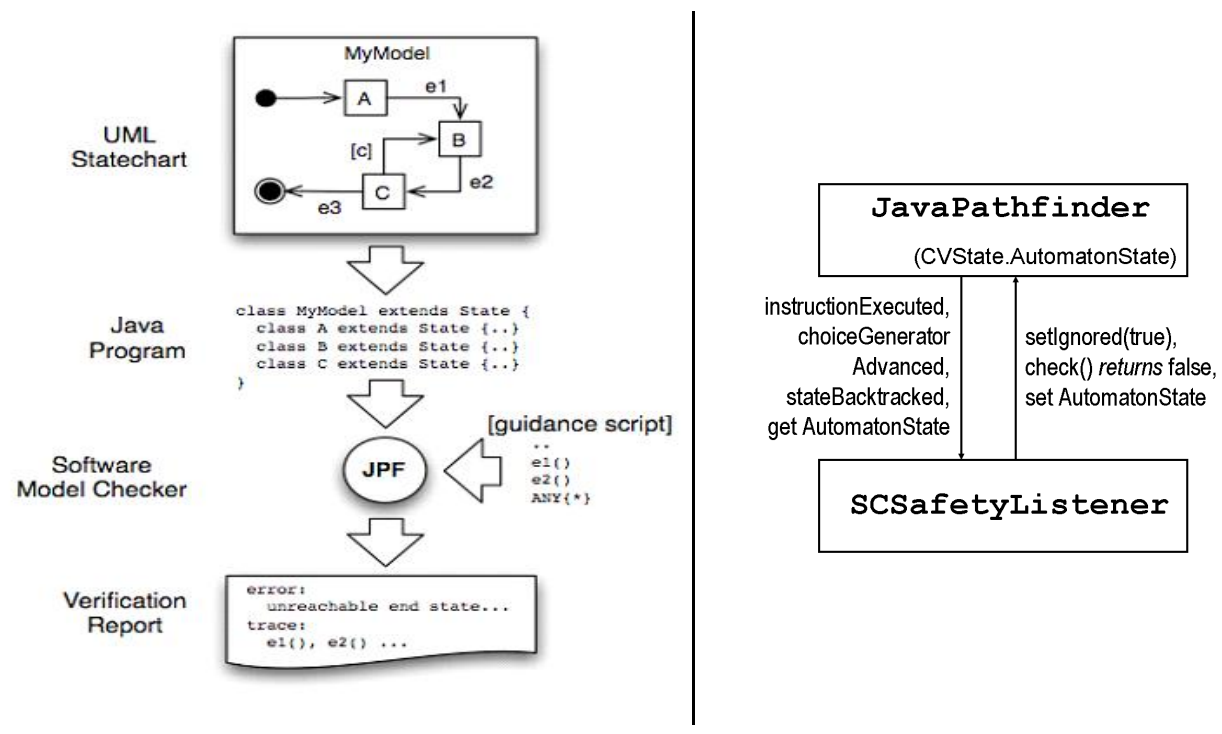

Fig. 4. Example illustrating JPF's UML extension (left) and JPF's listener (right)

user, which represents the input event sequences that can be provided by the external environment.

We have used the JPF statechart extension to implement our interface synthesis algorithms for components expressed in the JPF statechart framework. Note that we do not attempt to perform compositional reasoning for UML statecharts, which is a theoretical challenge that is beyond the scope of this work. Rather, we use UML statecharts, as supported by JPF, to represent finite state components with Labeled Transition System semantics. Therefore composition of components comes down to LTS composition, as described in Section 2. The interfaces that we generate are expressed as LTSs in the FSP notation [13].

\subsection{Assume-Guarantee Reasoning in JPF}

We have implemented assume-guarantee reasoning in JPF. As mentioned, components are given as UML statecharts (instances of class CVState). Both properties and assumptions are represented as finite state automata (instances of class gov.nasa.jpf.cv.SCSafetAutomaton).

Model checking using assumptions and properties has been implemented using JPF listeners (Figure 4(right)). A listener is essentially configured client code that is notified when certain events occur while JPF performs its search. The notified listener code can interact with JPF, e.g. a JPF "property" listener informs JPF if the property holds via the return value of its check() method.

Checking for both assumptions and properties is implemented with the gov.nasa.jpf.cv.SCSafetyListener class. On creation, a SCSafetyListener is associated with a finite state automaton $P$, which expresses the property or assumption to be used during model checking. Note that the state of a listener 
is not included in the state that JPF explores / stores during model checking. However, the state of the automaton $P$ needs to be part of the state space for correct state-space exploration and backtracking. We perform this by adding a static integer field of class CVState for the cv extension, which is set from within the listener.

An SCSafetyListener listens for and reacts to the following events:

- instructionExecuted: Signals to the listener that an instruction was executed by JPF. The listener reacts by invoking method advance (...) on the automaton $P$. Advancing the automaton corresponds to making a state transition, if the instruction that was executed corresponds to an action in the alphabet of the automaton. If a transition on an alphabet action is undefined from the current state, this is an illegal transition (corresponds to a transition to the error state $\pi$ ). For properties, this means that an error has occurred, so the result returned by the listener's check() method is false.

- choiceGeneratorAdvanced: Signals that the next statechart action is selected for execution. The reaction of the listener is to check whether this action would make $P$ transition to the error state if it were to be executed (this does not change the state of $P$ since the transition is not really executed yet). Reaching an error state in an assumptions means that the current path explored is not a valid path under this assumption and must therefore be ignored. The listener forces JPF to backtrack (by executing vm.getSystemState (). setIgnored (true)).

- stateBacktracked: When the model checker backtracks, then the automaton must backtrack accordingly.

For example, in order to check some property described as an automaton provided in some file Foo, we need to include the following arguments when running JPF's main class gov.nasa.jpf . JPF:

+ jpf.listener=.cv.SCSafetyListener

+safetyListener 1 . property= Foo

The first argument informs JPF that an SCSafetyListener will need to be notified of specific events, and the second one provides details for the listener, i.e., its unique id is " 1 ", it is of type property (as opposed to assumption), and the automaton associated with it is provided in file Foo (this may also include the full path to Foo).

\subsection{Interface Generation and Discharge}

The interface generation in JPF is implemented in the main class gov.nasa.jpf.tools.cv.ScRunCV. The user can customize the generation via the following arguments:

+assumption. alphabet=<actions $>$ defines the interface alphabet;

+assumption .outputFile $=\langle$ file name $>$ defines a file in which the generated interface is output. 


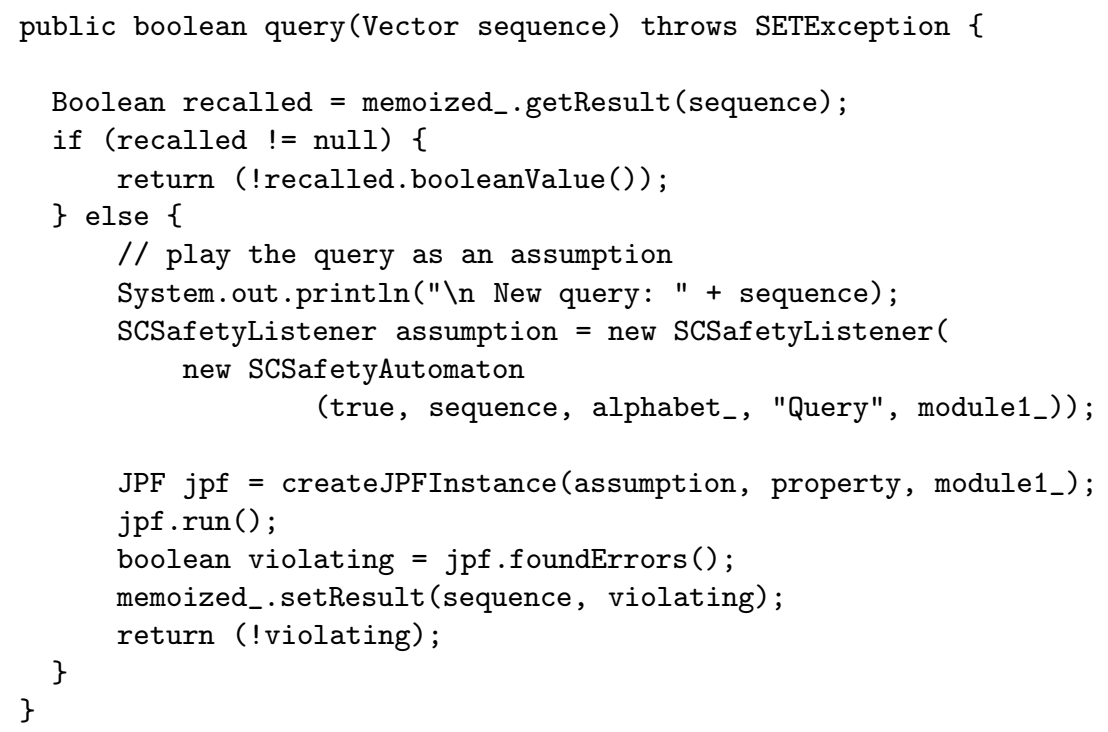

Fig. 5. Answering queries in SCModularTeacher

This allows for a generated interface to be used for subsequent reasoning, either as an assumption, or as a property. The format currently used for expressing the interface is the FSP language.

The main method of gov.nasa.jpf . tools.cv. ScRunCV creates an instance of class gov.nasa.jpf.tools.cv.SETLearner to carry out the learning of the interface; an associated instance of gov.nasa.jpf.tools.cv.SCModularTeacher serves as the teacher. Our learning algorithm implementation uses JPF to perform the model checking steps described in Section 3. JPF model checks individual components in the context of the universal environment. Listeners are added as necessary to reflect the work of the Teacher, which consists of answering Queries, and implementing Oracle 1 and Oracle 2 in order to answer conjectures, as described in more detail below.

Queries and Oracle 1. Queries and Oracle 1 are performed in a similar fashion because they are concerned with checking whether error states are reachable in the component, in the context of a particular sequence (for queries) or finite state automaton (for Oracle1). As illustrated in Figure 5, to respond to a query, a listener instance assumption is created with an associated automaton that reflects the particular sequence that is being queried. JPF is then invoked, together with the assumption listener. If JPF returns errors, the answer to the query is false, otherwise the answer is true. Oracle 1 works in a similar fashion, with the difference that it also returns a counterexample.

Oracle 2. Oracle 2 checks for permissiveness of a computed interface. It needs to work on the completed component, as described in Section 3 . This is a manual step that we intend to automate in the future. It similarly invokes JPF, 
but performs the search in the context of a specialized type of listener, the gov.nasa.jpf.cv.SCConformanceListener. Its aim is to detect the reachability of a $(\pi, o k)$ combination of states in the interface and component where the interface is in an error state, while the component is in an non-error state.

The gov.nasa.jpf.cv.SCConformanceListener listens for and reacts to the following events:

- executeInstruction: When the instruction about to be executed by JPF is an assertion violation, then it means that the component has entered an error state. Since such states are not targeted by the listener, it performs ti.skipInstruction(); vm.getSystemState(). setIgnored (true); .

The first command ensures that the exception is not processed by JPF, for efficiency. The second asks JPF to backtrack since this path cannot lead to the targeted combination of states.

- instructionExecuted: Similar to gov.nasa.jpf.cv.SCSafetyListener. When the automaton associated with the listener moves to an error state, the result returned by the $\operatorname{check}()$ method of the listener is set to false, since the component is in a legal state (illegal states are never reached since the listener advises JPF to backtrack when it reacts to executeInstruction events), while the interface is in an error state.

- stateBacktracked: Similar to gov.nasa.jpf.cv.SCSafetyListener.

As described in Section 3. when an $(\pi, o k)$ state is detected by the model checker, the counterexample leading to this state is queried, and if it is not a real counterexample, the model checker will backtrack. Since a query involved calling the model checker, this would involve nested model checker calls. To avoid such nesting, our implementation exploits a memoized table that is used by the learner to store results of previous queries. Oracle 2 checks for the reachability of $(\pi, o k)$ states in a loop. Whenever a counterexample is obtained by the model checker, then OraclE2 invokes a query on it. Each query stores its result in the memoized table.

Whenever a real counterexample is obtained, Oracle 2 exits the loop and reports the result to the learner. When a counterexample is spurious, then another iteration of the loop is entered. In this iteration, we wish to ensure that the model checker will not report the same spurious counterexample. We achieve this as follows. When a gov.nasa.jpf.cv.SCSafetyAutomaton is asked to advance in the context of a gov.nasa.jpf.cv.SCConformanceListener, if the automaton reaches an error state, it will get the path to this state from JPF. It will then check the memoized table to see if there is a result for the corresponding sequence stored there. If there is, and the result is true, then it means that this is a spurious counterexample, and it notifies JPF to backtrack. Therefore, we have implemented the nested model checking calls by consecutive calls to the model checker, where the information of spurious counterexamples is shared through the memoized table. 


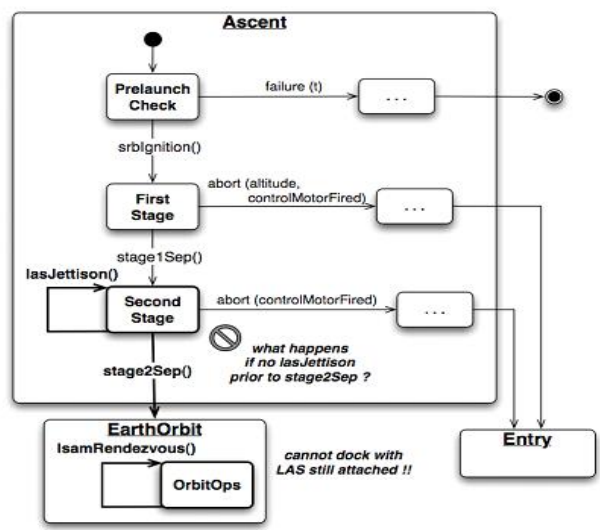

Fig. 6. Model of the Ascent and Earth Orbit flight phases of a spacecraft

Interface discharge. For compositional reasoning, one needs to also discharge the generated interface on the component environment. This can be performed by model checking the environment component in the presence of a gov.nasa.jpf.cv.SCSafetyListener using the interface as a property.

\section{Experience}

In order to evaluate our implementation, we used a statechart model of the Ascent and EarthOrbit flight phases of a space-craft (see Figure [6). The JAVA model is available with the JPF distribution under examples/jpfESAS. The UML statechart diagrams for the model are included in examples/jpfESAS. doc.

The model was created and used to demonstrate the features of the JPF UML statechart extension to our NASA mission customers. Several properties were analyzed on the model, and JPF returned violations for some of these properties. When the counterexamples obtained were analyzed, it was clear that some of the violations were spurious. The violations were related to the following properties:

- An event lsamRendezvous, which represents a docking maneuver with another spacecraft, fails if the LAS (launch abort system) is still attached to the spacecraft.

- Event tliBurn (trans-lunar interface burn takes spacecraft out of the earth orbit and gets it into transition to the moon) can only be invoked if EDS (Earth Departure Stage) rocket is available.

These violations were due to the fact that the universal environment was too general. The models had been created under the assumption that the use of the model respects some implicit flight rules. We decided to use our interface generation techniques to formalize the flight rules. More specifically, for each property, 


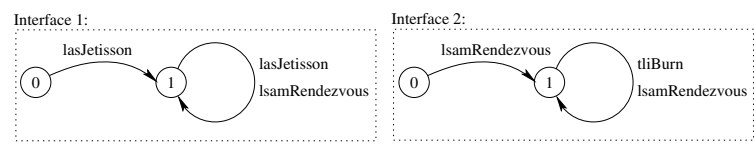

Fig. 7. Generated interface specifications

we generated a safe and permissive interface to eliminate its corresponding violations. To do this, we added a listener that eliminated all assertion violations that were not related to the targeted property, through the following arguments: + jpf. .listener=.tools. ChoiceTracker: .cv. AssertionFilteringListener +assertionfilter. include=<method_name>

These arguments specify that all assertion violations that occur outside the particular <method_name> will be ignored.

The generated interface specifications are illustrated in Figure 7 . The first one expresses the fact that the lsamRendezvous maneuvers cannot start before the las module of the spacecraft has jettisoned. According to the second one, it does not make sense to perform tliBurn prior to performing lsamRendezvous. These interfaces were inspected by the developer of the model that confirmed that they encode actual flight rules. Interface generation can therefore be used by developers to help them in the expression of the assumptions that their models encode. We note that other examples, including the input-output example from [6], are available with the JPF distribution.

\section{Conclusions}

We have proposed an algorithm for automatically synthesizing behavioral interface specifications for finite state software components. Our algorithm is the first iterative approach that is guaranteed to compute interfaces that are both safe and permissive, even in the presence of non-determinism in the visible behavior of a component. We have implemented our approach in the JavaPathfinder model checking framework for UML statechart components, and have obtained promising results from its application to several systems. The source code for the implementation and the examples is available through JPF's distribution.

In the future, we plan to investigate interface generation for methods with parameters. We have made some initial experiments using JPF's symbolic execution extension to generate values for parameters with infinite domains, and used these values to define finite interface alphabets related to their corresponding methods. We wish to pursue this direction further, and also plan to extend our results to generic Java components. For components that may be infinite-state, we will combine our approach with techniques such as predicate abstraction (similar to [1]). Finally, we plan to perform extensive evaluations of our approach.

\section{Acknowledgements}

We thank Peter Mehlitz and Suzette Person for helping with the implementation. 


\section{References}

1. Alur, R., Cerny, P., Madhusudan, P., Nam, W.: Synthesis of interface specifications for Java classes. In: Proceedings of POPL 2005, pp. 98-109 (2005)

2. Alur, R., Henzinger, T., Mang, F., Qadeer, S., Rajamani, S., Tasiran, S.: MOCHA: Modularity in Model Checking. In: Vardi, M.Y. (ed.) CAV 1998. LNCS, vol. 1427, pp. 521-525. Springer, Heidelberg (1998)

3. Ammons, G., Bodik, R., Larus, J.R.: Mining specifications. In: Proceedings of ACM POPL 2002, pp. 4-16 (2002)

4. Angluin, D.: Learning regular sets from queries and counterexamples. Information and Computation 75(2), 87-106 (1987)

5. Beyer, D., Henzinger, T.A., Singh, V.: Algorithms for Interface Synthesis. In: Damm, W., Hermanns, H. (eds.) CAV 2007. LNCS, vol. 4590, pp. 4-19. Springer, Heidelberg (2007)

6. Cobleigh, J.M., Giannakopoulou, D., Pasareanu, C.S.: Learning Assumptions for Compositional Verification. In: Garavel, H., Hatcliff, J. (eds.) TACAS 2003. LNCS, vol. 2619, pp. 331-346. Springer, Heidelberg (2003)

7. Flanagan, C., Freund, S.N., Qadeer, S.: Thread-Modular Verification for SharedMemory Programs. In: Le Métayer, D. (ed.) ESOP 2002. LNCS, vol. 2305, pp. 262-277. Springer, Heidelberg (2002)

8. Giannakopoulou, D., Pasareanu, C.S., Barringer, H.: Assumption Generation for Software Component Verification. In: Proceedings of ASE 2002, pp. 3-12. IEEE Computer Society, Los Alamitos (2002)

9. Groce, A., Peled, D., Yannakakis, M.: Adaptive Model Checking. In: Katoen, J.P., Stevens, P. (eds.) TACAS 2002. LNCS, vol. 2280, p. 357. Springer, Heidelberg (2002)

10. Henzinger, T.A., Jhala, R., Majumdar, R.: Permissive Interfaces. In: Proceedings of ESEC/SIGSOFT FSE 2005, pp. 31-40 (2005)

11. Java PathFinder, http://javapathfinder.sourceforge.net

12. Jones, C.B.: Specification and Design of (Parallel) Programs. In: Information Processing 1983: Proceedings of the IFIP 9th World Congress, IFIP, pp. 321-332. North Holland, Amsterdam (1983)

13. Magee, J., Kramer, J.: Concurrency: State Models \& Java Programs. John Wiley \& Sons, Chichester (1999)

14. Margaria, T., Raffelt, H., Steffen, B., Leucker, M.: The LearnLib in FMICS-jETI. In: Proceedings of ICECCS 2007 (2007)

15. Mehlitz, P.: Trust Your Model - Verifying Aerospace System Models with Java Pathfinder. In: IEEE/Aero (2008)

16. Pasareanu, C.S., Giannakopoulou, D., Gheorghiu Bobaru, M., Cobleigh, J.M., Barringer, H.: Learning to Divide-and-Conquer: Applying the L* Algorithm to Automate Assume-Guarantee Reasoning. In: FMSD (January 2008)

17. Pnueli, A.: In Transition from Global to Modular Temporal Reasoning about Programs. In: Logic and Models of Concurrent Systems, vol. 13, pp. 123-144 (1984)

18. Tkachuk, O., Dwyer, M.B.: Adapting side effects analysis for modular program model checking. In: Johansson, T. (ed.) FSE 2003. LNCS, vol. 2887, pp. 188-197. Springer, Heidelberg (2003)

19. Whaley, J., Martin, M.C., Lam, M.S.: Automatic extraction of object-oriented component interfaces. In: Proceedings of ISSTA 2002, pp. 218-228 (2002) 